\title{
Contrôle optimal par réduction de modèle POD et méthode à région de confiance du sillage laminaire d'un cylindre circulaire
}

\author{
Michel Bergmann ${ }^{\mathrm{a}}$ et Laurent Cordier $^{\mathrm{b}, \mathrm{c}}$ \\ LEMTA, UMR 7563 (CNRS-INPL-UHP), ENSEM, 2 avenue de la forêt de Haye, BP 160, 54504 Vandœuvre Cedex, France
}

Reçu le $1^{\text {er }}$ septembre 2006, accepté le 23 janvier 2007

\begin{abstract}
Résumé - L'objectif de cette étude est de minimiser, par rotation sinusoïdale, le coefficient de traînée moyen d'un cylindre circulaire en régime laminaire. Une procédure d'optimisation couplant modèle réduit par POD et méthode à région de confiance (TRPOD) est utilisée. Cette approche conduit à une réduction du coefficient de traînée de $30 \%$ pour un coût de calcul limité.
\end{abstract}

Mots clés : Contrôle optimal / réduction de modèle / POD / méthode à région de confiance / sillage / cylindre circulaire / traînée

\begin{abstract}
Optimal control of the circular cylinder wake in the laminar regime by POD reduced-order models and trust-region method. The objective of this study is the mean drag minimization under rotary control of the cylinder wake in the laminar regime. The optimization problem is solved by a procedure that couples POD Reduced-Order Models (POD ROM) and trust-region method (TRPOD). Finally, $30 \%$ of relative mean drag reduction is found for reduced numerical costs.
\end{abstract}

Key words: Optimal control / reduced-order model / POD / trust-region method / wake flow / circular cylinder / drag

\section{Introduction}

\subsection{De l'intérêt de la réduction de modèles en contrôle d'écoulement}

Ces dernières années, les industriels du domaine aéronautique et automobile ont apporté un regain d'intérêt au contrôle actif d'écoulements et à l'optimisation de formes aérodynamiques. Formellement, ces problèmes se ramènent à minimiser une fonction objectif $\mathcal{J}$ (coefficient de traînée, concentration de polluant, bruit émis, ...) en fonction de $n$ paramètres $\boldsymbol{c}=$ $\left(c_{1}, c_{2}, \ldots, c_{n}\right) \in \mathbb{R}^{n}$ (vitesse de soufflage/aspiration, flux de chaleur, paramètres de formes, ...) sous certaines contraintes (équations de Navier-Stokes, contraintes géométriques, ...). Grossièrement, les différentes méthodes

\footnotetext{
${ }^{a}$ Nouvelle adresse : MAB, UMR 5466, Université Bordeaux 1, 351 cours de la Libération, 33405 Talence Cedex, France

b Nouvelle adresse : LEA, UMR 6609, CEAT, 43 route de l'aérodrome, 86000 Poitiers, France

c Auteur correspondant :

Laurent.Cordier@univ-poitiers.fr
}

de résolution existantes peuvent être classées en deux catégories, les méthodes de type descente qui nécessitent au minimum une approximation du gradient de la fonction objectif, et les méthodes stochastiques dont le principe consiste à étudier l'évolution d'une population d'individus au cours de générations successives (algorithme génétique par exemple). Quelle que soit la méthode envisagée, les coûts de calcul liés à ces méthodes de résolution sont tellement importants qu'elles deviennent inadaptées aux applications du contrôle actif en boucle fermée, pour lesquelles le contrôleur a besoin de déterminer son action en temps réel.

Dans cet article, nous proposons de résoudre le problème d'optimisation associé au contrôle d'écoulement par une méthode de contrôle optimal dans laquelle les équations d'état ne sont plus constituées par les équations de Navier-Stokes mais par un modèle réduit de dynamique de l'écoulement contrôlé construit par POD (Proper Orthogonal Decomposition). Cette technique, introduite à l'origine en Turbulence [1] afin d'extraire les Structures Cohérentes, permet d'évaluer une base, optimale au sens de l'énergie, qui peut être utilisée par la suite pour construire par projection de Galerkin sur les 


\section{Nomenclature}

\begin{tabular}{|c|c|}
\hline$\Omega$ & Domaine spatial d'étude \\
\hline$e_{x}$ & Vecteur de norme unité orienté dans le sens de l'écoulement amont \\
\hline$e_{y}$ & $\begin{array}{l}\text { Vecteur de norme unité perpendiculaire à } \boldsymbol{e}_{x} \\
\left(\boldsymbol{e}_{x}, \boldsymbol{e}_{y}\right) \text { forment une base orthonormale directe }\end{array}$ \\
\hline $\boldsymbol{x}=(x, y)^{\mathrm{T}}$ & Vecteur position d'un point quelconque de $\Omega$ \\
\hline$\Gamma_{\mathrm{c}}$ & Frontière du cylindre \\
\hline $\boldsymbol{n}=\left(n_{x}, n_{y}\right)^{\mathrm{T}}$ & Normale extérieure de $\Gamma_{\mathrm{c}}$ (vecteur de norme unité) \\
\hline$R e$ & Nombre de Reynolds \\
\hline$U_{\infty}$ & Vitesse à l'infini amont en $\mathrm{m} . \mathrm{s}^{-1}$ \\
\hline$D$ & Diamètre du cylindre en $\mathrm{m}$ \\
\hline$\nu$ & Viscosité cinématique en $\mathrm{m}^{2} \cdot \mathrm{s}^{-1}$ \\
\hline$V_{\mathrm{T}}(t)$ & Vitesse tangentielle sur $\Gamma_{\mathrm{c}}$ en $\mathrm{m} . \mathrm{s}^{-1}$ \\
\hline$\gamma(t)$ & Loi de contrôle (sans dimension) \\
\hline$A$ & Amplitude de la loi de contrôle $\gamma$ \\
\hline$S t_{\mathrm{f}}$ & Nombre de Strouhal de la loi de contrôle $\gamma$ \\
\hline$u(\boldsymbol{x}, t)$ & Composante du vecteur vitesse selon $\boldsymbol{e}_{x}$ (sans dimension) \\
\hline$v(\boldsymbol{x}, t)$ & Composante du vecteur vitesse selon $\boldsymbol{e}_{y}$ (sans dimension) \\
\hline$p(\boldsymbol{x}, t)$ & Pression (sans dimension) \\
\hline $\boldsymbol{U}=(u, v, p)^{\mathrm{T}}$ & Variable d'état \\
\hline $\boldsymbol{U}_{\mathrm{m}}=\left(u_{\mathrm{m}}, v_{\mathrm{m}}, p_{\mathrm{m}}\right)^{\mathrm{T}}$ & Champ moyen \\
\hline $\boldsymbol{U}_{\mathrm{c}}=\left(u_{\mathrm{c}}, v_{\mathrm{c}}, p_{\mathrm{c}}\right)^{\mathrm{T}}$ & Mode de contrôle \\
\hline$\phi_{i}=\left(\phi_{i}^{u}, \phi_{i}^{v}, \phi_{i}^{p}\right)^{\mathrm{T}}$ & Vecteur propre POD d'ordre $i$ \\
\hline$\zeta_{\mathrm{NS}}$ & Variable d'état déterminée par le modèle Navier-Stokes \\
\hline$\zeta_{\mathrm{POD}}$ & Variable d'état déterminée par le modèle réduit POD \\
\hline$N_{\mathrm{POD}}$ & Nombre de modes POD \\
\hline$N_{\text {gal }}$ & Nombre de modes Galerkin \\
\hline$N_{\text {neq }}$ & Nombre de modes de non-équilibre \\
\hline$C_{\mathrm{D}}(t)$ & Coefficient de traînée \\
\hline$C_{\mathrm{L}}(t)$ & Coefficient de portance \\
\hline $\mathcal{C}_{\mathrm{D}}$ & Opérateur de traînée \\
\hline$T$ & Temps sur lequel est calculé le coefficient de traînée moyen \\
\hline$c^{(k)}$ & Contrôle à l'itéré $k$ de l'algorithme TRPOD \\
\hline$\Delta^{(k)}$ & Rayon de la région de confiance \\
\hline $\mathcal{J}^{(k)}$ ou $f\left(\boldsymbol{c}^{(k)}\right)$ & Fonction objectif réelle \\
\hline$\widehat{\mathcal{J}}^{(k)}$ ou $m^{(k)}\left(\boldsymbol{c}^{(k)}\right)$ & Fonction objectif modèle \\
\hline$\widehat{\boldsymbol{U}}^{\left[\mathrm{m}, \mathrm{c}, 1, \ldots, N_{\mathrm{gal}}\right]}$ & Estimation de la variable d'état $\boldsymbol{U}$ \\
\hline & Les modes utilisés sont indiqués dans le crochet $[\cdots]$ \\
\hline $\mathcal{B}_{i j}, \mathcal{C}_{i j k}, \mathcal{D}_{i}, \mathcal{E}_{i}, \mathcal{F}_{i j}, \mathcal{G}_{i}$ & Coefficients du modèle réduit POD \\
\hline$a_{i}(t)$ & Solution d'ordre $i$ du système d'état \\
\hline$\xi_{i}(t)$ & Solution d'ordre $i$ du système adjoint \\
\hline
\end{tabular}

équations du mouvement un modèle réduit de dynamique de l'écoulement contrôlé [2]. En dépit de l'optimalité énergétique de la base POD, il paraît cependant difficile de construire une fois pour toute, en début du processus d'optimisation, une base POD capable de décrire l'ensemble des écoulements susceptibles d'être rencontrés par le système au cours du processus d'optimisation (voir discussion menée en [3]). Une réactualisation de la base POD au cours du processus d'optimisation semble donc incontournable, la difficulté consistant à déterminer le moment où une nouvelle résolution des équations de Navier-Stokes est nécessaire pour évaluer une autre base POD. Par la suite, nous utiliserons un algorithme dénommé TRPOD pour Trust-Region POD [4] qui couple méthode d'optimisation à région de confiance et modèles réduits POD (voir Sect. 2).

\subsection{Configuration de l'étude}

Dans cette étude, nous cherchons à contrôler le sillage instationnaire existant en aval d'un cylindre circulaire (Fig. 1) pour un nombre de Reynolds $R e=200$ ( $R e=$ $\frac{U_{\infty} D}{\nu}$ avec $U_{\infty}$ vitesse à l'infini amont du cylindre, $D$ son diamètre et $\nu$, la viscosité cinématique du fluide). 


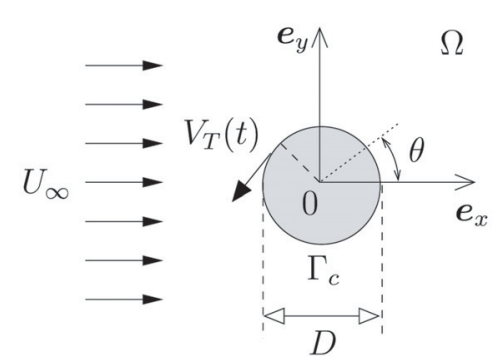

Fig. 1. Représentation schématique de notre configuration d'écoulement contrôlé modèle.

L'écoulement est considéré comme incompressible et le fluide est supposé visqueux et newtonien.

La dynamique d'un écoulement de sillage en régime laminaire est caractérisée par son nombre de Strouhal $S t$ défini comme $S t=\frac{f D}{U_{\infty}}$ où $f$ est la fréquence caractéristique du comportement périodique de l'écoulement (Fig. 7a). Le contrôle de l'écoulement est exercé par rotation instationnaire du cylindre autour de sa génératrice. Soit $V_{\mathrm{T}}$ la vitesse tangentielle du cylindre, le paramètre de contrôle du problème est $\gamma(t)=V_{\mathrm{T}}(t) / U_{\infty}$. Pour $\gamma=0$, l'écoulement sera dit non contrôlé. Dans la suite, nous recherchons la loi de contrôle $\gamma$ sous forme harmonique i.e. telle que :

$$
\gamma(t)=A \sin \left(2 \pi S t_{\mathrm{f}} t\right)
$$

où l'amplitude $A$ et le nombre de Strouhal de forçage $S t_{\mathrm{f}}$ correspondent aux deux degrés de liberté du contrôle.

\subsection{Définition de la fonction objectif}

Afin de simplifier les notations futures, on introduit l'opérateur de traînée $\mathcal{C}_{\mathrm{D}}$ comme :

$$
\begin{aligned}
\mathcal{C}_{\mathrm{D}} & : \mathbb{R}^{3} \longrightarrow \mathbb{R} \\
\boldsymbol{U} & \mapsto 2 \int_{\Gamma_{\mathrm{c}}}\left(p n_{x}-\frac{1}{R e} \frac{\partial u}{\partial x} n_{x}-\frac{1}{R e} \frac{\partial u}{\partial y} n_{y}\right) \mathrm{d} \Gamma=C_{\mathrm{D}}(t)
\end{aligned}
$$

où $\boldsymbol{U}=(u, v, p)^{\mathrm{T}}$ est le champ vectoriel correspondant aux variables de vitesse et de pression et $C_{\mathrm{D}}$, le coefficient de traînée instationnaire. L'objectif de l'étude est la minimisation du coefficient de traînée moyen de l'écoulement calculé sur un horizon de temps $T$ égal à quelques périodes de décrochement tourbillonnaire, soit :

$$
\left\langle C_{\mathrm{D}}(t)\right\rangle_{\mathrm{T}}=\frac{1}{T} \int_{0}^{\mathrm{T}} C_{\mathrm{D}}(t) \mathrm{d} t=\frac{1}{T} \int_{0}^{\mathrm{T}} \mathcal{C}_{\mathrm{D}}(\boldsymbol{U}) \mathrm{d} t
$$

\section{Optimisation par méthodes à région de confiance et modèles réduits $P O D$}

Dans cette section, seul le principe de la méthode sera exposé. Pour tous les détails et en particulier les preuves de convergence, le lecteur est prié de se référer à $[3,4]$.
L'objectif est de résoudre le problème d'optimisation :

$$
\min _{\boldsymbol{c} \in \mathbb{R}^{n}} \mathcal{J}\left(\boldsymbol{\zeta}_{\mathrm{NS}}(\boldsymbol{c}), \boldsymbol{c}\right)
$$

où $\mathcal{J}: \mathbb{R}^{m} \times \mathbb{R}^{n} \longrightarrow \mathbb{R}$ est la fonction objectif et où $\boldsymbol{\zeta}_{\mathrm{NS}}(\boldsymbol{c})$ et $c$ représentent respectivement les variables d'état obtenues par résolution numérique des équations du système et les variables de contrôle. L'indice NS signifie que les équations d'état qui relient les variables de contrôle $\boldsymbol{c}$ aux variables d'état sont les équations de Navier-Stokes. Il est possible, par résolution d'un système dynamique d'ordre réduit, de reconstruire ces variables par utilisation d'une base POD. En notant $\boldsymbol{\zeta}_{\mathrm{POD}}$ ces variables, le problème (2) peut être remplacé par une suite de sous-problèmes de la forme

$$
\min _{\boldsymbol{c} \in \mathbb{R}^{n}} \mathcal{J}\left(\boldsymbol{\zeta}_{\mathrm{POD}}(\boldsymbol{c}), \boldsymbol{c}\right)
$$

Or, le modèle réduit de dynamique construit par POD ne représente la dynamique de l'écoulement contrôlé que sur une région limitée de l'espace des paramètres de contrôle dite région de confiance. Posons $\Delta^{(k)}$ le rayon de la région de confiance et $\boldsymbol{c}^{(k)}$ les paramètres de contrôle obtenus pour un itéré $k$ de l'algorithme TRPOD. Pour évaluer la fonction $\mathcal{J}\left(\boldsymbol{\zeta}_{\mathrm{NS}}\left(\boldsymbol{c}^{(k)}\right), \boldsymbol{c}^{(k)}\right)$, il est nécessaire de déterminer les variables $\boldsymbol{\zeta}_{\mathrm{NS}}\left(\boldsymbol{c}^{(k)}\right)$. Ces variables sont obtenues par résolution des équations de Navier-Stokes. Lors de cette résolution, il est possible d'extraire des réalisations (snapshots) de l'écoulement contrôlé, et ainsi de générer une base POD $\left\{\phi_{i}\right\}_{i=1, \ldots, N_{\mathrm{POD}}}^{(k)}$. Cette base est utilisée pour construire un modèle d'ordre faible. L'intégration temporelle de ce modèle d'ordre faible permet de reconstruire les champs $\boldsymbol{\zeta}_{\mathrm{POD}}\left(\boldsymbol{c}^{(k)}\right)$, et donc d'évaluer la fonction $\mathcal{J}\left(\boldsymbol{\zeta}_{\mathrm{POD}}\left(\boldsymbol{c}^{(k)}\right), \boldsymbol{c}(k)\right)$. On définit ensuite la fonction

$$
\begin{aligned}
m^{(k)}\left(\boldsymbol{c}^{(k+1)}\right) & =m^{(k)}\left(\boldsymbol{c}^{(k)}+\boldsymbol{s}^{(k)}\right) \\
& =\widehat{\mathcal{J}}\left(\boldsymbol{\zeta}_{\mathrm{POD}}\left(\boldsymbol{c}^{(k)}+\boldsymbol{s}^{(k)}\right), \boldsymbol{c}^{(k)}+\boldsymbol{s}^{(k)}\right)
\end{aligned}
$$

comme fonction modèle de la fonction objectif

$$
\begin{aligned}
f\left(\boldsymbol{c}^{(k+1)}\right) & =f\left(\boldsymbol{c}^{(k)}+\boldsymbol{s}^{(k)}\right) \\
& =\mathcal{J}\left(\boldsymbol{\zeta}_{\mathrm{NS}}\left(\boldsymbol{c}^{(k)}+\boldsymbol{s}^{(k)}\right), \boldsymbol{c}^{(k)}+\boldsymbol{s}^{(k)}\right)
\end{aligned}
$$

à l'intérieur de la région de confiance $\left\|\boldsymbol{s}^{(k)}\right\| \leq \Delta^{(k)}$.

On est alors amené à résoudre le problème

$$
\min _{\boldsymbol{s} \in \mathbb{R}^{n}} m^{(k)}\left(\boldsymbol{c}^{(k)}+\boldsymbol{s}\right) \quad \text { sous les contraintes }\|\boldsymbol{s}\| \leq \Delta^{(k)}
$$

Il faut ensuite évaluer la performance des nouveaux paramètres de contrôle $\boldsymbol{c}^{(k+1)}=\boldsymbol{c}^{(k)}+\boldsymbol{s}^{(k)}$ obtenus par résolution du problème (3). On compare alors la décroissance réelle de la fonction objectif, $\operatorname{ared}^{(k)}=$ $f\left(\boldsymbol{c}^{(k)}+\boldsymbol{s}^{(k)}\right)-f\left(\boldsymbol{c}^{(k)}\right)$, à la décroissance prédite par la fonction modèle, $\operatorname{pred}^{(k)}=m^{(k)}\left(\boldsymbol{c}^{(k)}+\boldsymbol{s}^{(k)}\right)-m^{(k)}\left(\boldsymbol{c}^{(k)}\right)$. Si la décroissance de la fonction objectif est jugée bonne, en comparaison de celle obtenue par la fonction modèle, 


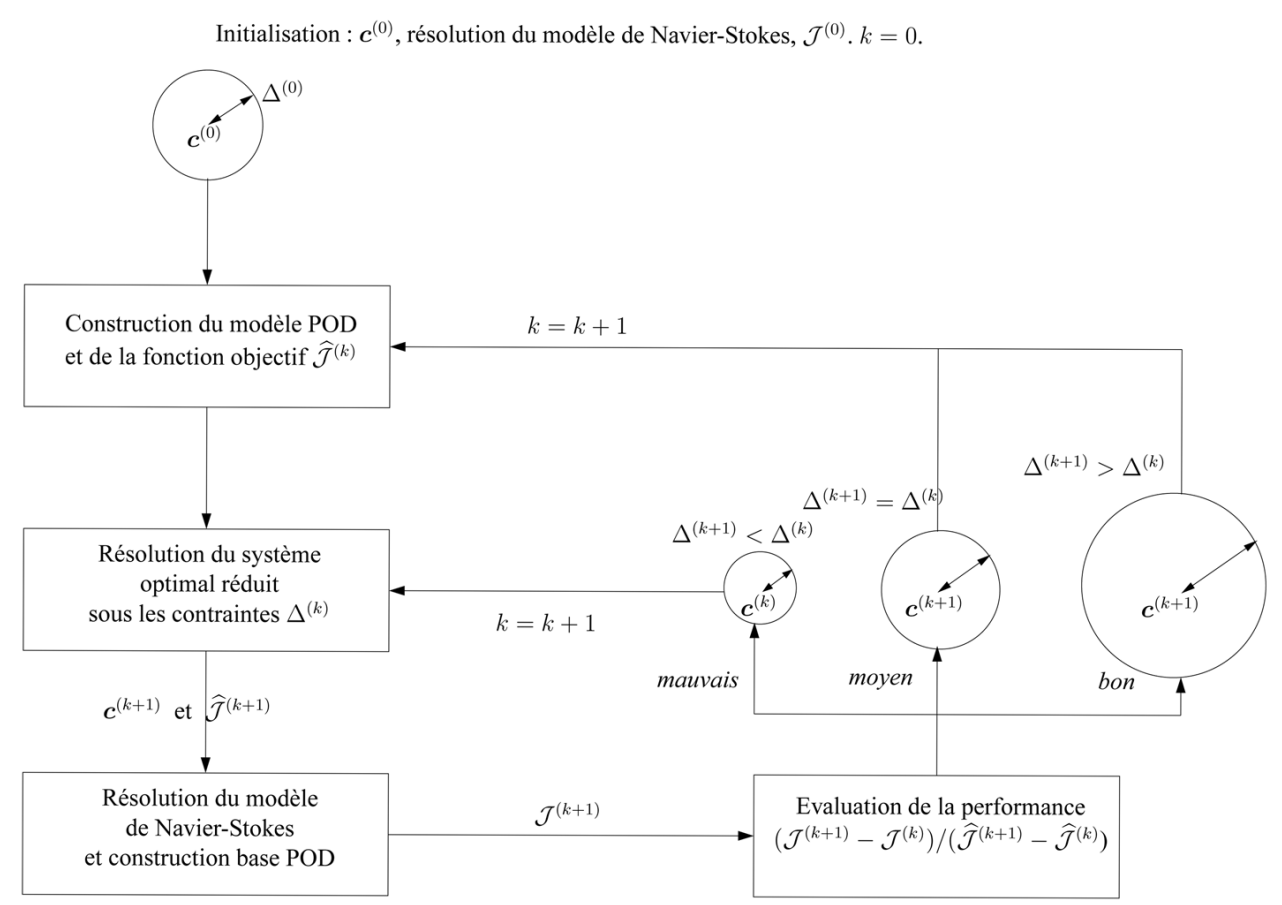

Fig. 2. Schématisation de la méthode à région de confiance.

le pas $\boldsymbol{c}^{(k+1)}=\boldsymbol{c}^{(k)}+\boldsymbol{s}^{(k)}$ est accepté et le rayon de confiance est augmenté à l'itéré suivant. Si la décroissance est jugée moyenne, le pas est accepté et le rayon de confiance est maintenu. Enfin, si la décroissance est jugée mauvaise, le pas est refusé et le rayon de confiance est diminué. Afin d'évaluer la valeur de la fonction objectif au point $\boldsymbol{c}^{(k+1)}, f\left(\boldsymbol{c}^{(k)}+\boldsymbol{s}^{(k)}\right)$, il est nécessaire de déterminer une nouvelle solution du modèle de précision, $\boldsymbol{\zeta}_{\mathrm{NS}}\left(\boldsymbol{c}^{(k)}+\boldsymbol{s}^{(k)}\right)$. Un autre jeu de réalisations, qui correspond au nouveau contrôle $\boldsymbol{c}^{(k+1)}$, est disponible. Ce jeu sert alors à construire un nouveau modèle réduit, qui est représentatif de la dynamique modifiée par application des paramètres de contrôle $\boldsymbol{c}^{(k+1)}$. L'ensemble de la procédure est résumé sur la figure 2.

\section{Application à la réduction de traînée d'un cylindre circulaire}

\subsection{Estimation robuste par modèle réduit POD du coefficient de traînée}

\subsubsection{Extension de la POD à la pression et méthode de la fonction de contrôle}

Pour mettre en œuvre l'algorithme TRPOD, présenté à la section 2, la variable $\boldsymbol{U}$ apparaissant dans la relation (1) doit pouvoir être évaluée soit par résolution du modèle de Navier-Stokes, soit par reconstruction à l'aide d'un modèle d'ordre réduit basé sur la POD. Pour que cela soit possible, nous avons étendu la base POD à la pression [3] et déterminé $\left\{\boldsymbol{\phi}_{i}=\left(\phi_{i}^{u}, \phi_{i}^{v}, \phi_{i}^{p}\right)^{\mathrm{T}}\right\}_{i=1}^{N_{\mathrm{POD}}}$. Par ailleurs, afin d'introduire de manière explicite la loi de commande $\gamma$ dans le modèle réduit POD, nous avons utilisé la méthode dite de la fonction de contrôle introduite dans [5]. Ainsi, toute réalisation $\boldsymbol{U}$ peut être approximée par le développement suivant :

$$
\begin{aligned}
\boldsymbol{U}(\boldsymbol{x}, t) & \simeq \widehat{\boldsymbol{U}}^{\left[\mathrm{m}, \mathrm{c}, 1, \ldots, N_{\mathrm{gal}}\right]} \\
& =\boldsymbol{U}_{\mathrm{m}}(\boldsymbol{x})+\gamma(\boldsymbol{c}, t) \boldsymbol{U}_{\mathrm{c}}(\boldsymbol{x})+\sum_{i=1}^{N_{\mathrm{gal}}} a_{i}(t) \boldsymbol{\phi}_{i}(\boldsymbol{x})
\end{aligned}
$$

où $N_{\text {gal }}$ est le nombre de modes Galerkin et où $\boldsymbol{U}_{\mathrm{m}}$ représente l'écoulement moyen. Enfin, le mode de contrôle $\boldsymbol{U}_{\mathrm{c}}$ est évalué comme une solution particulière des équations de Navier-Stokes, déterminée de telle manière que les fonctions propres soient homogènes sur le bord du domaine.

\subsubsection{Modes de non-équilibre}

Afin d'améliorer la robustesse du modèle réduit de dynamique, la base POD déterminée pour un paramètre de contrôle $\boldsymbol{c}$ donné, a été modifiée en y ajoutant $N_{\text {neq }}$ modes de non-équilibre, suivant en cela la procédure décrite dans [6]. Les modes POD de non-équilibre sont ajoutés à la base POD existante afin d'obtenir d'autres directions d'évolution que celles présentes initialement dans la base. Afin de conserver l'orthonormalité des fonctions de base POD, une procédure de Gram-Schmidt est utilisée. 
Les champs $\boldsymbol{U}$ peuvent alors être approximés sur la base propre POD par l'expression :

$$
\begin{aligned}
\widehat{\boldsymbol{U}}^{\left[\mathrm{c}, 0, \ldots, N_{\mathrm{gal}}+N_{\mathrm{neq}}\right]}(\boldsymbol{x}, t)=\gamma(\boldsymbol{c}, t) \boldsymbol{U}_{\mathrm{c}}(\boldsymbol{x}) \\
+\underbrace{\sum_{i=0}^{N_{\mathrm{gal}}} a_{i}(t) \boldsymbol{\phi}_{i}(\boldsymbol{x})}_{\text {modes POD Galerkin }}+\underbrace{\sum_{i=N_{\mathrm{gal}}+1}^{N_{\mathrm{gal}}+N_{\mathrm{neq}}} a_{i}(t) \boldsymbol{\phi}_{i}(\boldsymbol{x})}_{\text {modes de non-équilibre }}
\end{aligned}
$$

Le système dynamique POD d'ordre réduit, de dimension $N_{\text {gal }}+N_{\text {neq }}+1$, est alors de la forme [3] :

$$
\begin{aligned}
\frac{\mathrm{d} a_{i}(t)}{\mathrm{d} t}= & \sum_{j=0}^{N_{\text {gal }}+N_{\text {neq }}} \mathcal{B}_{i j} a_{j}(t) \\
& +\sum_{j=0}^{N_{\text {gal }}+N_{\text {neq }}} \sum_{k=0}^{N_{\text {gal }}+N_{\text {neq }}} \mathcal{C}_{i j k} a_{j}(t) a_{k}(t) \\
+\mathcal{D}_{i} \frac{\mathrm{d} \gamma}{\mathrm{d} t}+ & \left(\mathcal{E}_{i}+\sum_{j=0}^{N_{\text {gal }}+N_{\text {neq }}} \mathcal{F}_{i j} a_{j}(t)\right) \gamma(\boldsymbol{c}, t)+\mathcal{G}_{i} \gamma^{2}(\boldsymbol{c}, t)
\end{aligned}
$$

avec les conditions initiales suivantes :

$$
a_{i}(0)=\left(\boldsymbol{U}(\boldsymbol{x}, 0)-\gamma(\boldsymbol{c}, 0) \boldsymbol{U}_{\mathrm{c}}(\boldsymbol{x}), \phi_{i}(\boldsymbol{x})\right)
$$

On peut noter que le mode de contrôle $\boldsymbol{U}_{\mathrm{c}}$ étant prédéterminé, il n'est pas affecté par des changements de dynamique au cours d'une procédure de contrôle. Par ailleurs, le mode correspondant à l'écoulement moyen $\boldsymbol{U}_{\mathrm{m}}=a_{0} \phi_{0}$ n'est habituellement pas résolu par le système dynamique POD car il est constant en temps. Cependant, lorsque des modes de non-équilibre sont introduits, ce dernier peut être pris en compte dans le système dynamique car il peut avoir un régime transitoire, traduit par des échanges d'énergie avec les modes de non-équilibre.

La figure 3 représente de manière schématique l'utilisation d'un mode de non-équilibre pour modéliser la transition d'une dynamique quelconque notée I vers une autre dynamique notée II. Les écoulements moyens (mode $\phi_{0}$ ) et les fluctuations associés aux dynamiques I et II sont généralement différents. Il faut donc rajouter un vecteur de translation pouvant faire évoluer l'écoulement d'une dynamique à l'autre, au moins en moyenne. Ce vecteur de translation correspond à $\phi_{\text {neq }}^{\mathrm{I} \rightarrow \mathrm{II}}=\phi_{0}^{\mathrm{II}}-\phi_{0}^{\mathrm{I}}$. Si la dynamique correspondant à l'état I est connue, il est tout à fait possible que la dynamique correspondant à l'état II soit inconnue, notamment dans des procédures d'optimisation utilisant des modèles d'ordre faible. Il paraît donc nécessaire de rajouter des modes de translation traduisant d'autres directions de l'espace physique contrôlé, initialement non prises en compte dans les modes Galerkin. Il convient alors d'entourer la dynamique I par plusieurs dynamiques II, III, IV, etc. généralisant des directions non prises en compte dans le système POD initial (sans modes de translation). La figure 4 illustre,
I Espace physique contrôlé $I$

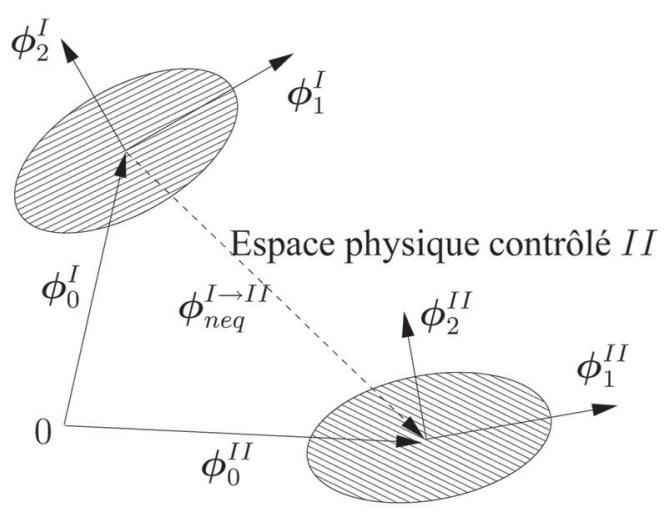

\section{Espace de contrôle}

Fig. 3. Représentation schématique d'une transition de dynamique par utilisation d'un mode moyen de non-équilibre. Pour des raisons de clarté, l'espace physique est réduit à trois directions : une direction pour l'écoulement moyen et deux directions pour les champs fluctuants.

en lignes de courant, l'allure de certains modes Galerkin et modes de non-équilibre pouvant être utilisés dans la décomposition (4).

\subsubsection{Fonction modèle et validation}

En notant $\boldsymbol{\zeta}_{\mathrm{NS}}(\boldsymbol{c})=\left(u_{\mathrm{NS}}, v_{\mathrm{NS}}, p_{\mathrm{NS}}\right)^{\mathrm{T}}$ et $\boldsymbol{\zeta}_{\mathrm{POD}}(\boldsymbol{c})=$ $\left(u_{\mathrm{POD}}, v_{\mathrm{POD}}, p_{\mathrm{POD}}\right)^{\mathrm{T}}$, la fonction objectif est

$$
f(\boldsymbol{c})=\mathcal{J}\left(\boldsymbol{\zeta}_{\mathrm{NS}}(\boldsymbol{c})\right)=\frac{1}{T} \int_{0}^{T} \mathcal{C}_{\mathrm{D}}\left(\boldsymbol{\zeta}_{\mathrm{NS}}(\boldsymbol{c})\right) \mathrm{d} t,
$$

et la fonction objectif modèle, introduite dans [3], est

$$
m^{(k)}(\boldsymbol{c})=\widehat{\mathcal{J}}\left(\boldsymbol{\zeta}_{\mathrm{POD}}(\boldsymbol{c})\right)=\frac{1}{T} \int_{0}^{\mathrm{T}} \sum_{i=0}^{N_{\mathrm{gal}}+N_{\mathrm{neq}}} a_{i}(t) N_{i} \mathrm{~d} t
$$

où $N_{i}=\mathcal{C}_{\mathrm{D}}\left(\phi_{i}\right)$.

Il est maintenant nécessaire de vérifier si la fonction objectif modèle est assez robuste pour représenter des comportements correspondant à des dynamiques différentes de celle utilisée pour construire le modèle d'ordre faible. La loi de contrôle $\gamma=A \sin \left(2 \pi S t_{\mathrm{f}} t\right)$ correspondant aux paramètres $A=2$ et $S t_{\mathrm{f}}=0,5$ est utilisée dans la décomposition (4) pour construire le système dynamique réduit (5). Ce système dynamique est ensuite intégré avec des paramètres de contrôle se situant dans le domaine $\mathcal{D}=\left\{1,5 \leq A \leq 2,5 ; 0,4 \leq S t_{\mathrm{f}} \leq 0,6\right\}$. Les valeurs de la fonction objectif modèle $\widehat{\mathcal{J}}$, qui correspondent à ces paramètres de contrôle peuvent en être déduites. La figure 5 représente les évolutions de la fonction objectif réelle $\mathcal{J}$ et de la fonction objectif modèle $\widehat{\mathcal{J}}$ lorsque 


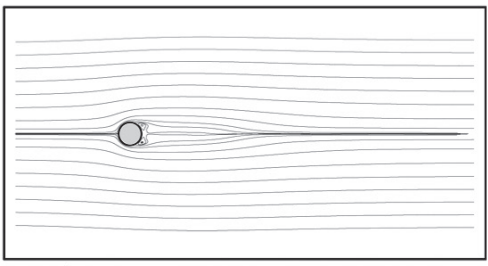

(a) Écoulement contrôlé $(I)$ moyen.

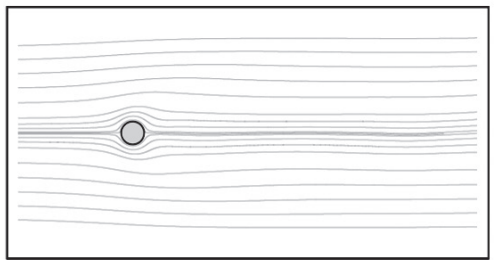

(d) Écoulement contrôlé (II) moyen.

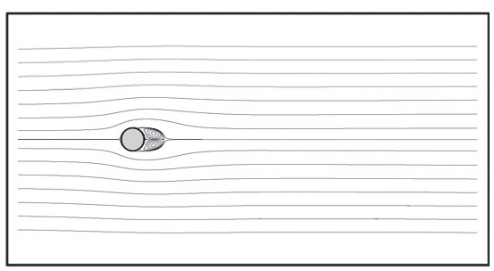

(g) Écoulement naturel (III) moyen.

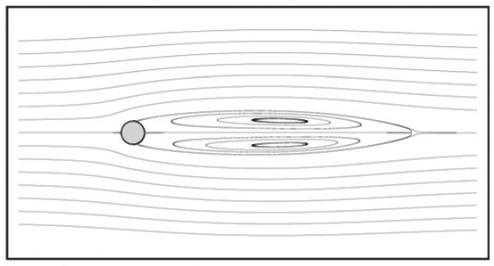

(j) Écoulement de base stationnaire instable $I V$.

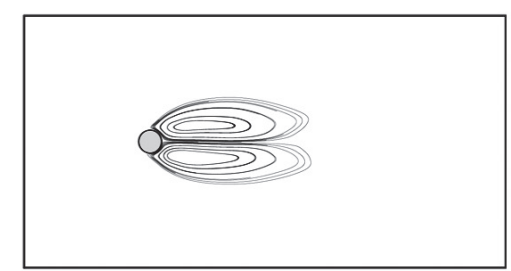

(m) Mode shift de $I$ vers $I I$.

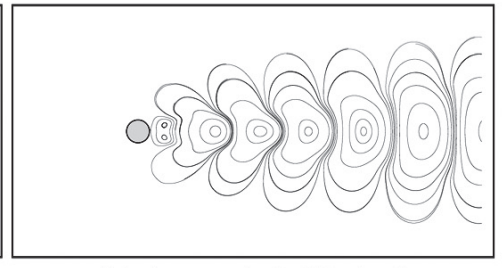

(b) ler mode POD de $I$.

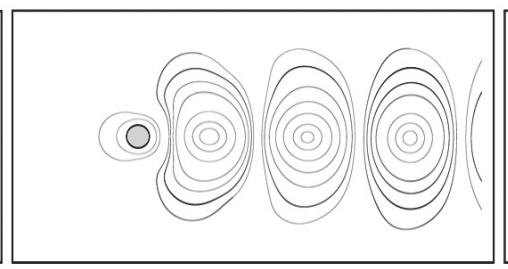

(e) ler mode POD de $I I$.

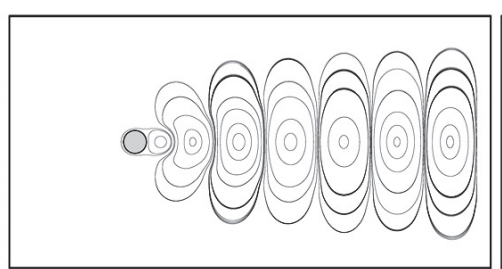

(h) 1er mode POD de $I I I$.

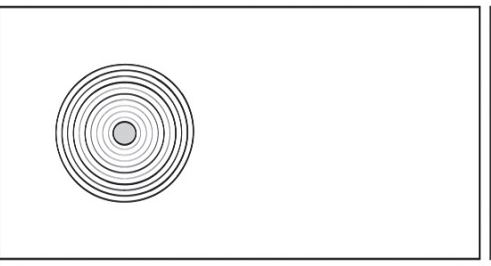

(k) Mode de contrôle.

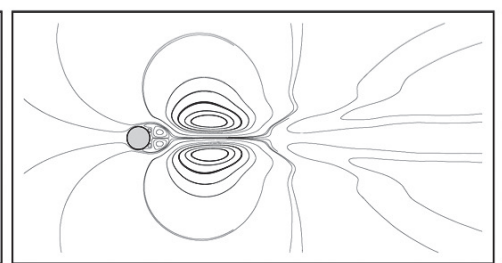

(n) Mode shift de $I$ vers $I I I$.

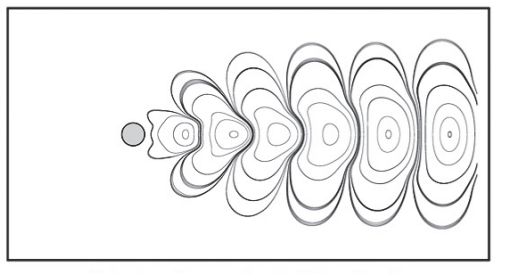

(c) 2nd mode POD de $I$.

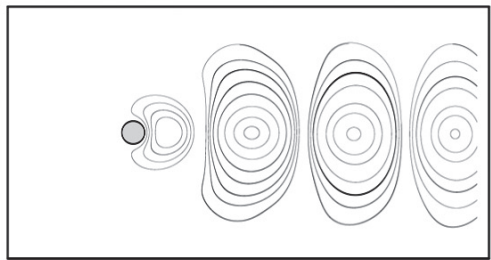

(f) 2nd mode POD de $I I$.

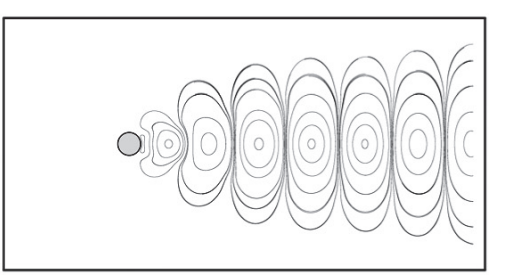

(i) 2nd mode POD de $I I I$.

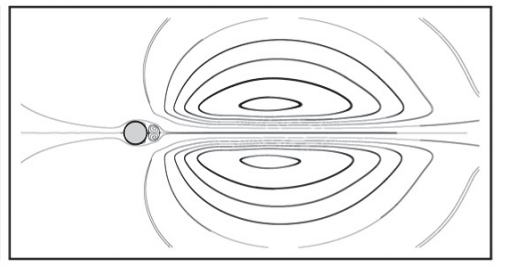

(1) Mode shift de $I I I$ vers $I V$.

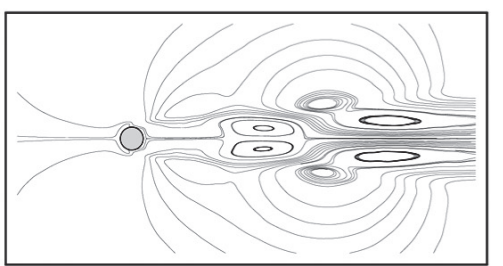

(o) Mode shift de $I$ vers $I V$.

Fig. 4. Représentation des modes Galerkin (a), (b) et (c), du mode de contrôle $\boldsymbol{u}_{\mathrm{c}}(\mathrm{k})$, et de modes de non-équilibre (m), (n), (o) pouvant être utilisés dans la décomposition dynamique (4). Tous les modes sont représentés par des lignes de courant à $R e=200$.

les paramètres de contrôle varient sur $\mathcal{D}$. On constate une très bonne concordance entre fonctions objectif et modèle, validant ainsi l'utilisation de la fonction modèle $\widehat{\mathcal{J}}$ en lieu et place de la fonction objectif $\mathcal{J}$.

\subsection{Formulation contrôle optimal}

Pour résoudre le sous-problème d'optimisation (3), le plus simple consiste à résoudre le système optimal construit à l'aide du modèle réduit de dynamique
POD. Ce système optimal est constitué des systèmes et équations suivantes [3] :

- système d'état (5)

- système adjoint

$$
\begin{aligned}
\frac{\mathrm{d} \xi_{i}(t)}{\mathrm{d} t}= & -\sum_{j=0}^{N_{\text {gal }}+N_{\text {neq }}}\left(\mathcal{B}_{j i}+\gamma(\boldsymbol{c}, t) \mathcal{F}_{j i}\right. \\
& \left.+\sum_{k=0}^{N_{\text {gal }}+N_{\text {neq }}}\left(\mathcal{C}_{j i k}+\mathcal{C}_{j k i}\right) a_{k}(t)\right) \xi_{j}(t)-\frac{1}{T} N_{i}
\end{aligned}
$$




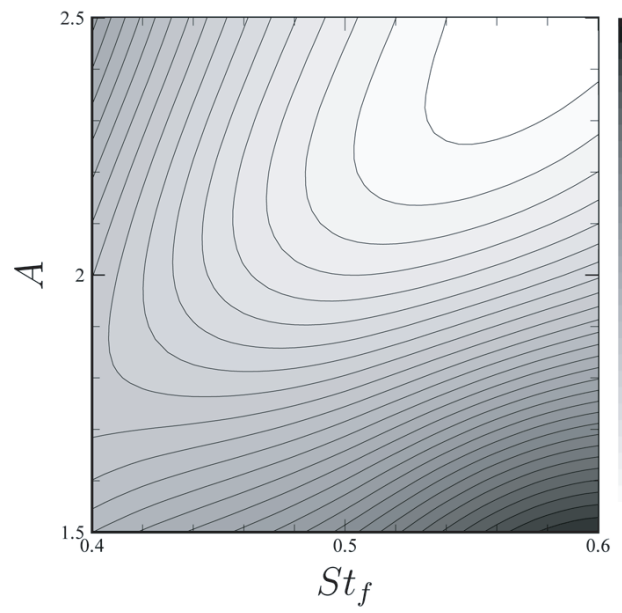

(a) Fonction objectif réelle $\mathcal{J}$

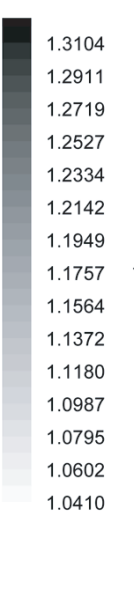

Fig. 5. Représentation des iso-valeurs des fonctions objectif réelle et modèle.

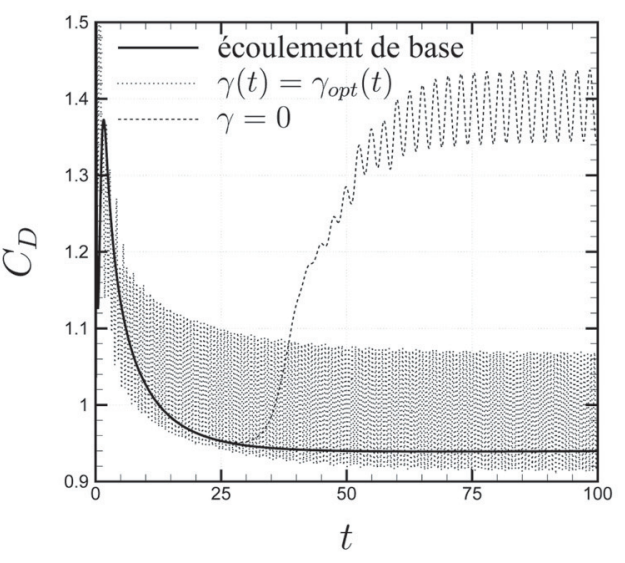

(a) Coefficient de traînée.

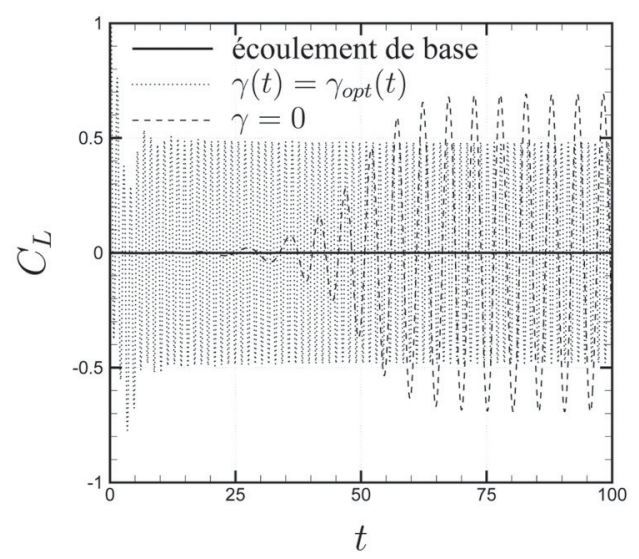

(b) Coefficient de portance.

Fig. 6. Comparaison de l'évolution temporelle des coefficients aérodynamiques dans le cas non contrôlé, dans le cas où le contrôle optimal est appliqué, et pour l'écoulement de base stationnaire instable.

muni des conditions terminales suivantes :

$$
\xi_{i}(T)=0
$$

- conditions d'optimalité

$$
\nabla_{\boldsymbol{c}} \mathcal{L}=\int_{0}^{\mathrm{T}}\left(\sum_{i=0}^{N_{\mathrm{gal}}+N_{\text {neq }}} \mathcal{L}_{i}\right) \nabla_{\boldsymbol{c}} \gamma \mathrm{d} t
$$

avec

$$
\mathcal{L}_{i}=-\frac{\mathrm{d} \xi_{i}}{\mathrm{~d} t} \mathcal{D}_{i}+\xi_{i}\left(\mathcal{E}_{i}+\sum_{j=0}^{N_{\text {gal }}+N_{\text {neq }}} \mathcal{F}_{i j} a_{j}+2 \gamma(\boldsymbol{c}, t) \mathcal{G}_{i}\right)
$$

Ce système peut être résolu à l'aide d'une méthode itérative décrite dans [3]. Lors de cette résolution, une contrainte supplémentaire, imposée par le rayon de la région de confiance, doit toutefois être prise en compte. Dans cette étude, les directions de descente sont construites à l'aide d'une méthode de gradient conjugué de type Fletcher-Reeves. La recherche linéaire est effectuée par une méthode d'Armijo à rebroussement, dans laquelle la longueur du pas initial, le long de chaque direction de descente, vérifie la contrainte imposée par la région de confiance.

\subsection{Résultats numériques et observations}

En utilisant la procédure TRPOD décrite précédemment, on détermine après convergence de l'algorithme que les paramètres de contrôle sont égaux à $A=4,25$ et $S t_{\mathrm{f}}=0,738$. Ces valeurs sont obtenues en une dizaine d'itérations (10 résolutions du modèle de NavierStokes). Ces résultats sont semblables à ceux prédits par expérimentation numérique. Les paramètres de contrôle, obtenus par méthodes à région de confiance, convergent vers les paramètres de contrôle optimaux, et ce, quels que soient les paramètres de contrôle initiaux utilisés. Ceci prouve la performance et la robustesse de la méthode d'optimisation décrite dans cet article. 


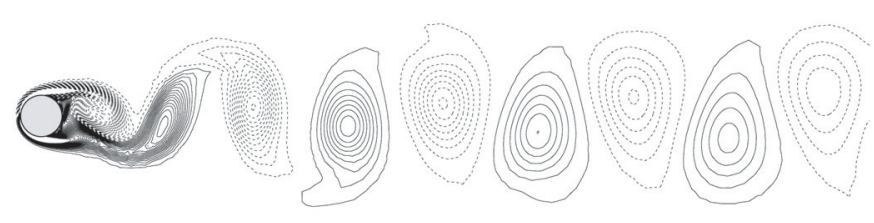

(a) Écoulement non contrôlé $(\gamma=0)$.

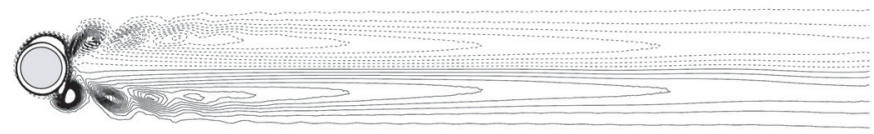

(b) Écoulement contrôlé $\gamma(t)=A \sin \left(2 \pi S t_{f} t\right), A=4,25$ et $S t_{f}=0,738$.

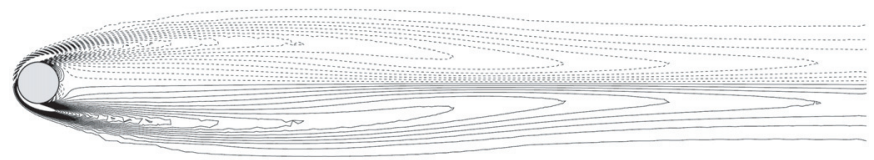

(c) Écoulement de base stationnaire instable.

Fig. 7. Représentation des iso-contours de vorticité pour un écoulement non contrôlé, pour un écoulement contrôlé avec les paramètres de contrôle optimaux $A=4,25$ et $S t=0,738$ et pour l'écoulement de base stationnaire instable.

Les figures 6(a) et (b) représentent respectivement l'évolution temporelle des coefficients de traînée et de portance, pour un écoulement non contrôlé et pour l'écoulement forcé en utilisant les valeurs des paramètres de contrôle optimaux déterminées par TRPOD. Ces résultats sont comparés avec le coefficient de traînée obtenu pour l'écoulement de base stationnaire instable. Cet écoulement de référence génère a priori le coefficient de traînée le plus faible pour la configuration étudiée [7]. Le coefficient de traînée moyen passe d'une valeur égale à 1,39 dans le cas non contrôlé à une valeur égale à 0,99 lorsque les paramètres de contrôle optimaux sont appliqués. Une réduction relative de $30 \%$ est obtenue. La valeur du coefficient de traînée pour l'écoulement contrôlé s'approche de la valeur du coefficient de traînée obtenue pour l'écoulement de base stationnaire instable $\left(C_{\mathrm{D}}=0,94\right)$. Par ailleurs, la dynamique de la vorticité pour l'écoulement contrôlé s'éloigne de la dynamique de l'écoulement non contrôlé pour s'approcher de la dynamique de l'écoulement de base stationnaire instable (voir Fig. 7).

\section{Conclusions}

Une procédure d'optimisation couplant méthodes à région de confiance et modèles réduits basés sur la POD a été mise en œuvre afin de minimiser le coefficient de traînée moyen du cylindre. Les paramètres de contrôle optimaux obtenus dans cette étude sont $A=$ 4,25 et $S t_{\mathrm{f}}=0,738$. La réduction relative du coefficient de traînée moyen est égale à 30 \%. Par ailleurs, l'utilisation de méthodes à région de confiance prouve mathématiquement la convergence des paramètres de contrôle obtenus par le modèle réduit vers les paramètres de contrôle optimaux du système Navier-Stokes. En outre, cette méthode permet de réduire considérablement les coûts de calcul. Ainsi, le système optimal obtenu en utilisant un modèle POD en lieu et place du modèle de NavierStokes comme système d'état, permet de stocker 1600 fois moins de valeurs numériques et est résolu environ 4 fois plus rapidement.

\section{Références}

[1] L. Cordier, M. Bergmann, Proper Orthogonal Decomposition: an overview, in Lecture series 2002-04 on postprocessing of experimental and numerical data, Von Kármán Institute for Fluid Dynamics, 2002

[2] L. Cordier, M. Bergmann, Two typical applications of POD: coherent structures eduction and reduced order modelling, in Lecture series 2002-04 on post-processing of experimental and numerical data, Von Kármán Institute for Fluid Dynamics, 2002

[3] M. Bergmann, Optimisation aérodynamique par réduction de modèle POD et contrôle optimal, Application au sillage laminaire d'un cylindre circulaire, thèse de doctorat, Institut National Polytechnique de Lorraine, Nancy, France, 2004

[4] M. Fahl, Trust-Region methods for flow control based on Reduced Order Modeling, thèse de doctorat, Université de Trier, Allemagne, 2000

[5] W.R. Graham, J. Peraire, K.T. Tang, Optimal Control of Vortex Shedding Using Low Order Models. Part 1, OpenLoop Model Development, Int. J. for Numer. Meth. Engrg. 44 (1999) 945-972

[6] B.R. Noack, K. Afanasiev, M. Morzyński, G. Tadmor, F. Thiele, A hierarchy of low-dimensional models for the transient and post-transient cylinder wake, J. Fluid Mech. 497 (2003) 335-363

[7] B. Protas, J.E. Wesfreid, Drag force in the open-loop control of the cylinder wake in the laminar regime, Phys. Fluids 14 (2002) 810-826 